\title{
WANGUN PAAJAH-AJAHAN BASA BALI SAJERONING ACARA SEKAR TAMAN RARE RING RADIO GENTA SWARA SAKTI BALI
}

\author{
Ni Luh Pt. Ellya NS ${ }^{1}$, IA Putu Purnami ${ }^{1}$, I Kt. Paramarta ${ }^{2}$ \\ Program Studi Pendidikan Bahasa Bali \\ Universitas Pendidikan Ganesha \\ Singaraja, Indonesia \\ e-mail: \{luhellya@gmail.com, dayupurnamiku@yahoo.com, \\ ketut.paramarta@undiksha.ac.id\}@undiksha.ac.id
}

\begin{abstract}
KUUB
Tetilikan puniki mahbahang indik (1) wangun paajah-ajahan basa Bali sajeroning acara Sekar Taman Rare miwah (2) paiketan wangun paajah-ajahan basa Bali sajeroning acara Sekar Taman Rare ring wangun paajah-ajahan sakadi inucap ring sekolah manut Taksonomi Bloom. Jejering tetilikan puniki inggih punika acara Sekar Taman Rare ring radio Genta Swara Sakti Bali. Panandang tetilikan puniki inggih punika wangun paajah-ajahan sajeroning acara Sekar Taman Rare. Sepat siku-siku sane kanggen sajeroning tetilikan puniki inggih punika (1) teori Taksonomi Bloom, (2) konsep tembang, (3) konsep anggah-ungguhing basa Bali, miwah (4) konsep nilai pendidikan karakter. Kramaning tetilik sane kanggen marupa kramaning dokumentasi miwah kramaning sadu wicara. Data tureksa sane kamargiang inggih punika pangresikan data, panyorohan data, pangwedaran data, miwah panyutetan. Pikolih tetilikan puniki marupa (1) wangun paajah-ajahan basa Bali sajeroning acara Sekar Taman Rare sane kapaiketang ring tetujon pendidikan manut Taksonomi Bloom inggih punika saking wangun kognitif, afektif, miwah psikomotor. (2) Paiketan wangun paajah-ajahan sajeroning acara Sekar Taman Rare ring wangun paajah-ajahan sakadi inucap ring sekolah minakadi paiketan sajeroning materi paajah-ajahan sane kadasarin antuk wangun kognitif, paiketan sajeroning nilai pendidikan karakter sane kadasarin antuk wangun afektif, miwah paiketan sajeroning kawagedan matembang miwah mabebaosan sane kadasarin antuk wangun psikomotor.
\end{abstract}

Kruna Jejaton: basa Bali, wangun paajah-ajahan

\begin{abstract}
This study aims to explain (1) a balinesse language learning pattern in Sekar Taman Rare show and (2) a balinesse language learning pattern relationship in Sekar Taman Rare show with such a learning pattern at school based on Taxonomi Bloom. The subject of this study is Sekar Taman Rare show on Genta Swara Sakti Bali radio. The object of this studi is Balinese language learning pattern in Sekar Taman Rare show. The theories used in this study are (1) Taksonomi Bloom theory, (2) the tembang concept, (3) the anggah-ungguhing basa Bali concept, and (4) the concept. The method that used in this study are documentation and interview method. Data analysis in this study are data reduction, data classification, data interpretation, and conclusion. The result of this study are (1) a Balinese language learning pattern in Sekar Taman Rare show is measured in cognitive, affective, and psychomotor aspects. (2) A balinesse language learning pattern relationship in Sekar Taman Rare show with such a learning pattern at school of relationships in learning materials covered in cognitive aspects, the relationship in the value education character reviewed from affective aspects, and the relationship of the matembang and mabebaosan skills based on psychomotor aspects.
\end{abstract}

Keywords: Balinesse language, learning pattern

\section{PURWAKA}

Basa Bali mabuat pisan majeng ring krama miwah budaya Bali. Basa Bali kabaos mabuat antuk kawentenannyane pinaka basa kapertama saha pinaka basa ibu sane kapolihang tur kaplajahin saking alit. Basa Bali taler pinaka sarana mabebaosan antuk krama Baline, sakadi mabebaosan ring kulawarga, banjar, adat, 
sajeroning paruman, miwah ri kala upacara yadnya. Samaliha, basa Bali taler pinaka sarana mlajahin sakancan budaya Baline sane sampun kaloktah kantos ka dura negara, minakadi lontar-lontar, tradisi adat, miwah sakancan upacara-upacara sane kalaksanayang ring Bali. Cutetnyane, sane pinih mautama inggih punika basa Bali pinaka kasujatian krama miwah budaya Baline.

Kautaman basa Bali pinaka kasujatian krama miwah budaya Bali ngawinang pemerintah urati pisan ring kawentenan miwah pelestarian basa Bali. Sajeroning UUD 1945 (Bab XV, Pasal 36) mungguh sakadi ring sor puniki.

"Bahasa-bahasa daerah yang
masih dipakai sebagai alat
perhubungan yang hidup dan dibina
oleh masyarakat pemakainya dihargai
dan dipelihara oleh Negara; karena
bahasa-bahasa itu adalah bagian
daripada kebudayaan Indonesia yang
hidup."

Bebaosan inucap nyantenang uratian saking pemerintah Indonesia ring basa daerah sakadi basa Bali. Aturanaturan sane tiosan inggih punika Peraturan Daerah (Perda) Provinsi Bali Nomor 3 tahun 1992 tentang Bahasa, Aksara, dan Sastra Bali taler sampun nyantenang pisan kawigunan saha kautaman basa Bali pinaka basa ibu sane patut kalestariang.

Utsaha-utsaha sane kalaksanayang antuk pemerintah Bali sane pinih anyar inggih punika ngamedalang Peraturan Gubernur Nomor 80 tahun 2018 tentang Perlindungan dan Penggunaan Bahasa, Aksara, dan Sastra Bali serta Penyelenggaraan Bulan Bahasa Bali. Ring pergub punika sampun tatas kaunggahang genah miwah galah sapatutnyane nganggen basa Bali pinaka krama Bali. Mabebaosan ring kulawarga, sajeroning parikrama agama, adat, miwah budaya Bali saha sajeroning ngicen utawi ngamedalang informasi majeng ring krama Bali antuk instansi pemerintahan wiadin swasta anggen nampingin basa Indonesia. Lembaga Bahasa, Aksara dan Sastra Bali taler sampun kawangun tur kawentenang sajeroning pergub punika mangda wenten lembaga khusus sane setata nguratiang panglimbak saha pelestarian basa Bali.

Utsaha sane tiosan inggih punika ngwentenang penyuluh basa Bali ring soang-soang desa sane wenten ring Bali. Swadharma penyuluh basa Bali ring soang-soang desa minakadi nampingin alit-alit miwah para yowana sajeroning nyastra Bali, sakadi ngicen tuntunan mlajah basa Bali. Pemerintah taler sampun nglaksanayang parikrama sakadi Pesta Kesenian Bali (PKB) pinaka jalaran nglestariang basa, aksara, miwah sastra Bali sane dagingnyane wenten pacentokan masatua Bali, mapidarta, makekawin, mageguritan, nyurat aksara Bali ring lontar, miwah sane tiosan. Pacentokan-pacentokan sakadi utsawa dharmagita, pacentokan macecimpedan, dolanan taler sampun kamargiang pinaka uratian saking pemerintah inggihan pelestarian basa Bali.

Akeh pisan program-program sane kalaksanayang antuk pemerintah Bali sajeroning nglestariang basa Bali. Sadurung wenten peraturan-peraturan pemerintah Bali indik pelestarian basa Bali, media massa sane wenten ring Bali sampun madue galah indik siaran mabasa Bali miwah budaya Bali. Indike puniki sairing ring panampen Jannet Holmes (1992) indik kawigunan media massa pinaka silih sinunggil pemertahanan basa daerah sakadi ring sor puniki.

"institutional support from domains such as education, law, administration, religion, and the media can make a difference between the success and failure of maintaining a minority group language".

Bebaosan inucap nyantenang pemertahanan basa daerah utaminyane basa Bali kaiusin antuk akehnyane media sane urati ring pelestarian basa inucap ring pasiakraman.

Media massa kapalih dados kalih, inggih punika media cetak miwah elektronik. Media cetak inggih punika wangun media massa sane marupa cetakan (printing) sane prasida kawacen. Soroh media cetak marupa surat kabar (koran) miwah majalah. Media elektronik inggih punika wangun media massa sane 
mapiranti antuk energy elektromekanis. Soroh media elektronik marupa media audiovisual inggih punika televisi miwah audio inggih punika radio. Ketahnyane, media massa ngunggahang gatra-gatra miwah acara-acara sane wenten sajeroning genah media inucap. Sakadi media massa inggihan media cetak miwah elektronik sane wenten ring Bali, sampun pastika ngunggahang acara miwah gatragatra sane mapaiketan ring krama miwah budaya Bali.

Media cetak ring Bali sampun wenten duk warsa 1924 kakawitin antuk mijilnyane majalah Shanti Adnyana sane kaobah dados Bali Adnyana ring Singaraja (Putra, 2010:72). Salanturnyane mijil majalah Surya Kanta, Canang Sari, Buratwangi, Kulkul miwah Bali Post. Media-media inucap ngunggahang gatragatra miwah sastra mabasa Bali saking sastrawan-sastrawan sane sampun kaloktah ring jagat Bali, sakewanten sajeroning panglimbaknyane wantah Bali Post sane kantun wenten kantos mangkin. Bali Post sane sampun wenten duk warsa 1948 puniki ngamijilang rubrik khusus nyabran Redite ngawit 20 Agustus 2006 sane kawastanin Bali Orti (Putra, 2010:110). Rubrik puniki madaging orti, satua cutet, satua masambung, puisi Bali Anyar, puisi Bali Purwa (sekar alit), miwah sane tiosan sane akehnyane petang kaca nyabran awuku.

Media massa salanturnyane inggih punika media audio visual (televisi). Media audio visual sakadi BaliTV (2002), TVRI Bali (1978) miwah Dewata TV (2007) taler urati ring pelestarian basa Bali. Media puniki sampun nyayagayang galah indik program mabasa Bali sajeroning TVnyane. Program indik orti mabasa Bali wenten Gatra Bali ring TVRI miwah Orti Bali ring BaliTV. Program indik tembang pop Bali wenten Dedalu ring TVRI miwah KLIP Bali ring BaliTV. Program sane tiosan inggih punika Masatua miwah Nyastra Bali ring TVRI saha BBQ (Banjar Bali Quiz) miwah Samatra Artis Bali ring BaliTV. Acara tiosan sane kaunggahang ring Dewata TV inggih punika Pentas Dewata sane kasenengin antuk kramane ring desa-desa.
Media massa sane kaping untat inggih punika media audio. Media massa marupa audio inggih punika radio sane wenten ring Bali akeh pisan, inggihan radio pemerintah wiadin swasta. $R R I$ Denpasar (88,6 FM), RRI Singaraja (108,0 FM), Radio Gema Merdeka (97,7 FM), Radio Genta Swara Sakti Bali (96,1 FM), Radio Citra Bali (98,7 FM), Singaraja FM $(92,0)$, Radio Pesona Bali (105,4 FM), Radio Guntur (104,6 FM) miwah sane tiosan. Program-program sane kaunggahang antuk radio inucap minakadi orti mabasa Bali miwah tembang-tembang Pop Bali. Wenten taler program-program khusus indik magegitayan minakadi makekawin miwah mageguritan sakadi Sudang Lepet Jukut Undis (SLJU) miwah Penglipur Sore ring RRI Singaraja. Sinalih tunggil acara sane sayuakti nutdut kayun inggih punika acara Sekar Taman Rare.

Acara Sekar Taman Rare inggih punika silih sinunggil program ring Radio Genta Swara Sakti Bali. Acara puniki kaunggahang nyabran rahina Redite ring galah 8 semeng kantos 10 tengai. Pamiletnyane inggih punika alit-alit ring wewidangan Denpasar, Badung, Tabanan, Gianyar, Klungkung, Jembrana, Bangli, miwah ring wewidangan Singaraja. Pramusiarnyane inggih punika anak istri sane ketah kasambat "mbok gek". Acara puniki wantah acara on air sane kaunggahang mangda pamiletnyane prasida nelpon miwah nunas tembang saha magatra nganggen basa Bali majeng ring pamilet sane tiosan. Ring galah tiosan, pramusiar taler ngwentenang segmen ngundang anak alit sane berprestasi sajeroning basa miwah budaya Bali, sakadi alit-alit sane dados anggota Alit Pewaris Bali Dwipa utawi APBD.

Yening selehin ring radio-radio sane tiosan, acara hiburan sane kaunggahang wantah indik tembang-tembang pop Bali kemanten. Nenten wenten sane ngunggahang program sakadi acara Sekar Taman Rare puniki, sane wantah katujuang majeng ring alit-alit. Wantah acara puniki sane urati majeng ring alit-alit Baline sajeroning nglestariang basa miwah budaya Bali. Sumeken pisan radio Genta Bali ngunggahang acara sakadi 
puniki cihna uratian ipun ring panglimbak basa Bali wantah ageng pisan. Asapunika taler indik galah acara puniki, inggih punika ring rahina Redite, sampun sinah pisan nenten pacang ngusik galah alitalite mlajah utawi masekolah. Tios ring pinaka hiburan ri kala Redite, acara puniki taler pinaka genah mlajah basa miwah budaya Bali antuk alit-alit sane setata mirengang acara puniki.

Makudang-kudang kaluihan sane kabaosang ring ajeng pinaka cihna paajah-ajahan prasida kamargiang nenten nginutin genah miwah galah sane formal. Sajeroning UU No. 20 Tahun 2003 Pasal 13 Ayat (1) kaunggahang genah pendidikan wenten tiga, inggih punika pendidikan formal, non-formal, miwah informal. Pendidikan formal pinaka pendidikan sane kamargiang antuk sekolah-sekolah sane madue undagan ngawit saking pendidikan dasar, pendidikan menengah, kantos pendidikan tinggi. Pendidikan non-formal pinaka pendidikan sane kamargiang nganutin wangun miwah undagan sane kawangun antuk sang sane ngamargiang pendidikan puniki, sakadi kursus, bimbingan belajar, miwah sane tiosan. Pendidikan informal inggih punika pendidikan sane kalaksanayang antuk mlajah mandiri tur bertanggungjawab sane kamargiang ring kulawarga miwah ring wewidangan tiosan. Indike puniki mapaiketan ring parikrama paajah-ajahan sane kamargiang ring acara Sekar Taman Rare nganutin pendidikan informal.

Daging-daging acara sajeroning acara Sekar Taman Rare puniki sakadi sekar rare, tembang pop anak-anak, puisi Bali Anyar, pupuh utawi macapat saha satua Bali pinaka daging paplajahan basa Bali ring sekolah. Mapaiketan ring Taksonomi Bloom, daging-daging paplajahan inucap ngranjing ring wangun kognitif duaning kawentenannyane pinaka pangweruhan majeng ring alit-alite. Uratian miwah rasa seneng alit-alite ri kala ngamiletin acara puniki pinaka cihna kawentenan wangun afektif sane kaunggahang sajeroning acara puniki. Yening selehin indik wangun psikomotomyane, kawagedan miwah rasa bangga ri kala alit-alite prasida matembang utawi mabasa Bali pinaka cinna sane becik. Punika mawinan ceciren-ceciren inucap nyantenang kawentenan wangun paajah-ajahan sajeroning acara puniki.

Ceciren sane tiosan inggih punika ri kala acara punika kamargiang. Ri kala acara sampun kakawitin tur sampun wenten pamilet sane nyarengin, pramusiar pacang nguncarang pangastungkara $\mathrm{Om}$ Swastyastu. Yening wenten pamilet sane nenten nyawis pangastungkara punika, pramusiar pacang nganikain pamilet sane nyarengin mangda nyawis antuk pangastungkara. Ring galah tiosan, pramusiar taler pinaka guru pengajian sane ngicen tuntunan ri kala pamilet sane nyarengin punika kantun kimud utawi kantun iwang sajeroning mabasa Bali miwah ri kala matembang. Sampun sinah pisan kawentenan pula-pali pangajahajahan basa Bali sajeroning acara Sekar taman Rare puniki sane minayang acara puniki ring acara tiosan sane wenten ring media massa, inggihan ring radio.

Acara puniki taler sampun nyinahang media pembelajaran majeng ring alit-alite ngawit mangkin, inggih punika paplajahan sane sampun ngwigunayang teknologi miwah media massa. Telepon sane kaanggen on air pinaka pirantinyane, miwah radio pinaka media massa inggian pendukung paajahajahan sane kalaksanayang ring acara puniki. Indike puniki pacang mawiguna pisan santukan alit-alite sampun uning genah mlajah punika boya ja ring sekolah kemanten nanging ring media massa sane masarana antuk teknologi sane wenten ring jero taler prasida kanggen mlajah, utamannyane mlajahin basa Bali. Indike puniki taler nyaratang kawigunan media massa nenten wantah pinaka genah ngrereh hiburan utawi genah malilacita kemanten.

Acara Sekar Taman Rare puniki sinah pisan kawigunan saha kawentenannyane pinaka genah mlajahin basa Bali inggihan basa miwah sastranyane antuk wangun paajah-ajahan sane matiosan ring wangun paajah-ajahan ring sekolah. Paajah-ajahan basa Bali sane kaunggahang nganggen sarana tembang-tembang Bali miwah dagiang 
paplajahan sane sampun kamanggehang ring sekolah-sekolah pinaka budaya krama Baline. Punika sane ngawinang tetilikan indik Wangun Paajah-Ajahan Basa Bali Sajeroning Acara Sekar Taman Rare Ring Radio Genta Swara Sakti Bali patut tur mabuat pisan kalaksanayang.

Kawentenan acara Sekar Taman Rare ring radio Genta Swara Sakti Bali sampun kaunggahang sakadi ring ajeng. Maduluran antuk bebaosane punika, panilik ngamanggihin makudang-kudang pikobet sane prasida katilikin sajeroning acara inucap. Pikobet-pikobet sane kapanggihin sakadi tembang pop anakanak, puisi Bali Anyar, parikrama masatua Bali, paiketan pamilet sareng pramusiar, kawigunan acara punika majeng ring krama Bali, miwah sane tiosan. Akeh pisan pikobet sane prasida katilikin sajeroning acara punika, sakewanten pikobet sane pacang katilikin inggih punika wantah indik paajah-ajahan basa Bali saha paiketannyane ring pendidikan formal.

Pikobet sane pacang katilikin sampun kajantenang ring ajeng inggih punika indik paajah-ajahan basa Bali saha paiketannyane ring pendidikan formal. Sajeroning ngamargiang tetilikan puniki, pikobet sane pacang katilikin kawatesin wantah sajeroning wangun paajah-ajahan basa Bali sane wenten ring acara Sekar Taman Rare saha wangun paajah-ajahan basa Bali inucap sane kamargiang irika. Nenten minayang utawi ngimbangang acara sane kamargiang punika ring pendidikan formal.

Bantang pikobet tetilikan (1) sapunapi wangun paajah-ajahan basa Bali sajeroning acara Sekar Taman Rare ring radio Genta Swara Sakti Bali miwah (2) sapunapi paiketan wangun paajah-ajahan basa Bali sajeroning acara Sekar Taman Rare ring wangun paajah-ajahan sakadi inucap ring sekolah manut Taksonomi Bloom.

Tetujon tetilikan puniki nlatarang (1) wangun paajah-ajahan basa Bali sajeroning acara Sekar Taman Rare ring radio Genta Swara Sakti Bali miwah (2) paiketan wangun paajah-ajahan basa Bali sajeroning acara Sekar Taman Rare ring wangun paajah-ajahan sakadi inucap ring sekolah manut Taksonomi Bloom.

Tetujon tetilikan punika kaarsa mangda madue kakalih kawigunan makadi (1) kawigunan pamucuk (teoretis) miwah (2) kawigunan panglimbak (praktis). Kawigunan pamucuk mapaiketan ring tata titi utawi sepat sikusiku sajeroning pangajahan basa Bali miwah kawigunan panglimbak mapaiketan ring sang sane prasida ngulati pikolih tetilikan puniki sakadi kabaosang ring sor puniki. Kawigunan pamucuk (teoritis) inggih punika kawigunan sane prasida kaanggen sepat siku-siku ri kala nglimbakang pangweruhan indik paajahajahan basa Bali. Kawigunan pamucuk puniki nyaratang mangda pikolih tetilikan puniki prasida ngaturang kaweruhan sajeroning nglimbakang sepat-siku-siku indik paajah-ajahan basa Bali miwah nglimbakang piranti paajah-ajahan basa Bali sajeroning aguron-aguron basa Bali.

Kawigunan panglimbak inggih punika kawigunan majeng ring sang sane prasida ngulati pikolih tetilikan puniki. Kawigunan puniki nyaratang mangda pikolih tetilikan puniki salanturnyane prasida mawiguna, pamekasnyane majeng ring paajah-ajahan basa Bali ring pakraman, paajah-ajahan basa Bali ring media massa, miwah paajah-ajahan basa Bali ring pendidikan formal. Kawentenan kawigunan panglimbak punika sajangkepnyane kabahbahang sakadi ring sor puniki. (a) Majeng ring paajah-ajahan basa Bali ring pakraman. Pikolih tetilikan puniki prasida pinaka piuning tur sesuluh ri kala ngamargiang paajah-ajahan basa Bali majeng ring alit-alite inggihan ring kekuwub kulawarga, dadia, banjar, miwah desa pakraman. (b) Majeng ring paajahajahan basa Bali ring media massa. Pikolih tetilikan puniki prasida pinaka piuning tur sesuluh sajeroning makarya acara utawi program siaran sane pateh antuk konsep acara sane matiosan. (c) Majeng ring paajah-ajahan basa Bali ring pendidikan formal. Pikolih tetilikan puniki prasida pinaka piuning majeng ring (1) pemerintah mangda wenten uratian sajeroning acara-acara sakadi puniki tur prasida nglimbakang kurikulum sane mapaiketan ring paajah-ajahan basa Bali. 
(2) Majeng ring sekolah, ngawit saking pendidikan usia dini kantos menengah mangda pikolih tetilikan puniki prasida kaanggen sesuluh ri kala ngamargiang paajah-ajahan basa Bali majeng ring sisiasisiane. (3) Majeng ring lembaga pendidikan tinggi, utaminnyane Program Studi Pendidikan Bahasa Bali Universitas Pendidikan Ganesha (UNDIKSHA) mangda pikolih tetilikan puniki prasida kaanggen jalaran mulat sarira sajeroning mata kuliah sane kamargiang ring perkuliahan mangda kadagingin kurikulum sane wenten ring pendidikan dasar.

\section{KRAMANING TETILIK}

Kramaning tetilikan inggih punika tata cara sajeroning ngamargiang tetilikan (Hikmat, 2011: 35). Bebaosan punika nyantenang, kramaning tetilikan inggih punika tata cara sajeroning ngamolihang, ngwangun, miwah ngringkupang data sajeroning tetilikan. (1) Wangun miwah Soroh Tetilikan. Wangun tetilikan inggih punika rerawatan miwah tata cara sane pacang kamargiang sajeroning tetilikan. Wangun tetilikan sane kamargiang indik wangun paajah-ajahan basa Bali sajeroning acara Sekar Taman Rare inggih punika wangun kualitatif santukan data tetilikan puniki marupa wicara sane mapaiketan ring parindikan manusa ring pakaramanipun.

Soroh tetilikan inggih punika rerawatan tata cara sane kaanggen sajeroning tetilikan (Sukmadinata, 2010:52). Soroh tetilikan indik wangun paajah-ajahan basa Bali sajeroning acara Sekar Taman Rare inggih punika soroh fenomenologis sane ngunggahang pikolih tetilikan saking wicara indik wangun paajah-ajahan basa Bali marupa bebaosan ring sesuratan. (2) Genah tetilikan. Genah tetilikan inggih punika genah sajeroning ngamargiang tetilikan sane madue ceciren wenten jejering, genah, miwah parikrama sane pacang katureksain. Genah tetilikan sane kamargiang puniki kakepah dados kalih, inggih punika ring genah stasiun radio puniki miwah ring jangkauan frekuensi utawi coverage area radio inucap. Stasiun radio punika kaanggen genah ngamargiang sadu wicara majeng ring sang sane pacang katakenang (informan). Jangkauan frekuensi utawi coverage area punika kaanggen genah miarsayang wangun paajah-ajahan basa Bali sane kamargiang irika. (3) Soroh miwah pawiwit data. Soroh data inggih punika ceciren utawi kahanan data sajeroning tetilikan. Soroh data sane kaanggen sajeroning tetilikan puniki wenten kalih, inggih punika data utama (primer) miwah data (sekunder). Pawiwit data inggih punika jejering genah data punika kapolihang sane marupa jadma, genah, wiadin simbol (Arikunto, 2006:129). Pawiwit data primer saking siaran miwah rekaman acara Sekar Taman Rare inucap sane suenyane kirang langkung abulan (4 episode). Pawiwit data sekunder saking sadu wicara majeng ring sang sane mapaiketan ring acara punika (informan kunci). (4) kramaning mupulang data sane kanggen marupa kramaning dokumentasi miwah sadu wicara. (5) Data tureksa sane kamargiang marupa pangresikan data, panyorohan data, pangwedaran data, miwah panyutetan.

\section{PIKOLIH MIWAH TETEPASAN}

Ring adyaya kaping pat puniki pacang kabahbahang indik pikolih miwah tetepasan tetilikan. Indike puniki mapaiketan sajeroning bantang pikobet sane nyihnayang indik (1) wangun paajahajahan basa Bali sajeroning acara Sekar Taman Rare ring radio Genta Swara Sakti Bali saha (2) paiketan wangun paajahajahan basa Bali inucap ring sekolah manut Taksonomi Bloom. Bebaosan inucap kajangkepin antuk implikasi sane midartayang indik paiketan miwah pikenoh daging tetilikan puniki majeng ring kawentenan mata kuliah ring Program Studi Pendidikan Bahasa Bali UNDIKSHA.
(1)
Wangun
paajah-ajahan basa Bali kaunggahang manut pikolih data sane sampun kaambil tur kasorohang salami asasih (petang episode). Pikolih data sane kaunggahang puniki marupa paajah-ajahan basa Bali sane wenten sajeroning acara Sekar Taman Rare sane kapaiketang ring tetujon pendidikan manut Taksonomi Bloom, inggihan ring (1) wangun kognitif, (2) wangun afektif, miwah (3) wangun psikomotor. Data sane 
kanggen marupa bebaosan pramusiar (Pr) miwah pamilet $(\mathrm{Pa})$ sajeroning acara Sekar Taman Rare inucap.

Manut pikolih tetilikan, kapanggihin paajah-ajahan basa Bali sane nganutin tetujon pendidikan minakadi wangun kognitif, afektif miwah psikomotor manut Taksonomi Bloom. Sajeroning wangun kognitif pinaka tetujon paajah-ajahan sane nyaratang indik kaweruhan kapanggihin paajah-ajahan tembang Bali miwah anggah-ungguhing basa Bali. Sajeroning wangun afektif sane nyaratang indik parilaksana (sikap) kapanggihin paajahajahan sane nyihnayang parilaksana sane becik saha mapaiketan ring pendidikan karakter. Sajeroning wangun psikomotor pinaka wangun paajah-ajahan sane nyaratang indik kawagedan utawi katrampilan kapanggihin kawagedan pamilet sajeroning matembang miwah mabebaosan nganggen basa Bali.

Wangun paajah-ajahan sane kaunggahang puniki ngranjing ring teori Taksonomi Bloom sane ngunggahang indik tetujon pendidikan (Sunarti miwah Rahmawati, 2014: 15). Sajeroning Taksonomi Bloom kaunggahang tiga tetujon pendidikan inggih punika wangun kognitif, wangun afektif, miwah wangun psikomotor. Makatetiga wangun puniki nenten prsida kapasahang santukan madue paiketan sane rumaket. Sakadi sane kaunggahang ring ajeng, kaweruhan sane kapolihang sajeroning wangun kognitif inggihan tembang Bali miwah anggah-ungguhing basa Bali sampun pastika pacang madue kawagedan utawi katrampilan sajeroning matembang miwah mabebaosan nganggen basa Bali. Asapunika taler sajeroning parilaksananyane ri kala matembang miwah mabebaosan nganggen basa Bali.

(2) Manut Taksonomi Bloom, wenten tiga wangun sane pinaka tetujon pendidikan, inggih punika wangun kognitif, wangun afektif, miwah wangun psikomotor. Wangun kognitif mapaiketan ring kaweruhan, wangun afektif mapaiketan ring parilaksana, miwah wangun psikomotor mapaiketan ring kawagedan utawi katrampilan. Mapaiketan ring tetiga wangun puniki, prasida kabahbahang paiketan wangun paajah-ajahan basa Bali sajeroning acara Sekar Taman Rare ring wangun paajahajahan sakadi inucap ring sekolah. Paiketan sane kapanggihin inggih punika (1) paiketan sajeroning materi paajahajahan sane kadasarin antuk wangun kognitif. (2) Paiketan sajeroning nilai pendidikan karakter sane kadasarin antuk wangun afektif miwah (3) paiketan sajeroning kawagedan matembang miwah mabebaosan sane kadasarin antuk wangun pasikomotor.

Paiketan wangun paajah-ajahan basa Bali sajeroning acara Sekar Taman Rare miwah ring sekolah prasida kasinahang antuk materi paajahajahannyane. Sajeroning acara Sekar Taman Rare kapanggihin paajah-ajahan tembang Bali inggihan Sekar Rare miwah Sekar Alit saha paajah-ajahan anggahungguhing basa Bali sakadi sane sampun kabahbahang ring bantang pikobet sane kapertama. Paajah-ajahan tembang Bali inggihan Sekar Rare miwah Sekar Alit saha paajah-ajahan anggah-ungguhing basa Bali ring sekolah kaunggahang ring silabus sane sampun wenten ring ajeng.

$$
\text { Sajeroning silabus punika }
$$

kasinahang paajah-ajahan Sekar Rare miwah Sekar Alit wenten ring kelas I miwah kelas II. Indike puniki kasinahang ring kompetensi dasar (KD) 3.2 ngeninin indik nguningin tembang Bali miwah KD 3.5 ngeninin indik tata cara nembangang pupuh Mijil miwah pupuh Pucung. Sekar Rare inggihan gending rare sane kanggen inggih punika tembang "Putri Cening Ayu". Sekar Alit sane kanggen materi paajahajahan inggih punika Pupuh Mijil miwah Pupuh Pucung. Indike puniki mapaiketan ring paajah-ajahan tembang Bali sane wenten ring acara Sekar Taman Rare santukan sajeroning paajah-ajahan Sekar Rarenyane wenten pamilet sane matembang "Putri Cening Ayu". Paajahajahan Sekar Alitnyane taler madue paiketan santukan sajeroning acara Sekar Taman Rare wenten pamilet sane nembangang pupuh Mijil. Indike puniki sampun nyihnayang sajeroning materi paajah-ajahan sane wenten ring acara Sekar Taman Rare sairing ring paajahajahan sane kalaksanayang ring sekolah. 
Paiketan sajeroning nilai pendidikan karakter puniki kasinahang antuk bebaosan sane wenten sajeroning acara Sekar Taman Rare miwah KI 1 saha KI 2 sane ngranjing ring wangun afektif sajeroning paajah-ajahan. Paajah-ajahan puniki kabaos mapaiketan sajeroning nilai pendidikan karakter santukan paajahajahan basa Bali sane mangkin kadasarin antuk K13 sane setata madasar antuk pendidikan karakter. Sajeroning acara Sekar Taman Rare, kapikolihang nilai pendidikan karakter sane wenten mawilang pitu (7) nilai inggih punika nilai religius, mandiri, ingin tahu, tanggung jawab, peduli lingkungan, cinta tanah air, miwah menghargai prestasi. Nilai religius puniki mapaiketan ring $\mathrm{KI} 1$ sane nyaratang indik rasa bakti sajeroning ngamargiang krama agama sane kasungkemin. Kasinahang antuk parilaksana pamilet ri kala ngawitin mabebaosan setata nguncarang Om Swastyastu.

Nilai mandiri, ingin tahu, tanggung jawab, peduli lingkungan, cinta tanah air, miwah menghargai prestasi puniki mapaiketan ring $\mathrm{KI} 2$ sane nyaratang indik parilaksana sisia sajeroning paajahajahan miwah sajeroning rasa bakti ring rerama miwah guru pengajian. Paiketan puniki nyihnayang wangun paajah-ajahan basa Bali sane wenten sajeroning acara Sekar Taman Rare ring wangun paajahajahan sakadi inucap ring sekolah madue wangun sane pateh kadasarin antuk paiketan nilai pendidikan karakternyane sane ngranjing ring wangun afektif sajeroning Taksonomi Bloom.

Paiketan puniki kaunggahang santukan wangun paajah-ajahan basa Bali sane ngranjing ring wangun psikomotor marupa kawagedan sajeroning matembang miwah mabebaosan nganggen basa Bali. Indike puniki sampun kabuktiang antuk wangun sane pateh sajeroning paajah-ajahan ring sekolah inggih punika sajeroning kompetensi inti miwah kompetensi dasar sane pinaka dasar sajeroning ngamargiang paajahajahan.

PAMUPUT
Ringkesan tetilikan puniki indik (1) bantang pikobet tetilikan inggih punika wangun paajah-ajahan basa Bali sajeroning acara Sekar Taman Rare ring radio Genta Swara Sakti Bali saha paiketannyane ring wangun paajah-ajahan sakadi inucap ring sekolah manut Taksonomi Bloom. (2) Tetujon tetilikan puniki kaanutang ring bantang pikobet sane kaunggahang. (3) Kawigunan tetilikan puniki wenten kalih, inggih punika kawigunan pamucuk miwah kawigunan panglimbak. (4) Sepat siku-siku sane kanggen inggih punika teori Taksonomi Bloom, konsep tembang, konsep anggahungguhing basa Bali, miwah konsep nilai pendidikan karakter. (5) Kramaning tetilik sane kanggen marupa kramaning dokumentasi miwah sadu wicara. (6) pikolih tetilikan puniki marupa Wangun paajah-ajahan basa Bali sane kapaiketang ring tetujon pendidikan manut Taksonomi Bloom kapanggihin wangun paajahajahan sane madasar antuk wangun kognitif marupa paajah-ajahan tembang Bali inggihan Sekar Rare miwah Sekar Alit sane mawilang pat belas tembang. Wangun paajah-ajahan sane madasar antuk wangun afektif marupa paajahajahan sajeroning nilai pendidikan karakter. Wangun paajah-ajahan sane madasar antuk wangun psikomotor marupa kawagedan sajeroning matembang Bali inggihan Sekar Rare miwah Sekar Alit saha mabebaosan nganutin anggah-ungguhing basa Bali sane patut.

Paiketan wangun paajah-ajahan basa Bali sajeroning acara Sekar Taman Rare ring wangun paajah-ajahan sakadi inucap ring sekolah manut Taksonomi Bloom kapaiketang madasar antuk silabus paajah-ajahan basa Bali ring SD. Paiketan sane kapanggihin marupa paiketan sajeroning materi paajah-ajahan, paiketan sajeroning nilai pendidikan karakter, miwah paiketan sajeroning kawagedan matembang miwah mabebaosan. Paiketan sajeroning materi paajah-ajahan puniki kadasarin antuk wangun kognitif, paiketan sajeroning nilai pendidikan karakter kadasarin antuk wangun afektif, miwah paiketan sajeroning kawagedan 
matembang miwah mabebaosan kadasarin antuk wangun psikomotor.

Malarapan antuk pikolih miwah tetepasan sane kabahbahang, prasida katingkesang sakadi ring sor. Wangun paajah-ajahan basa Bali sajeroning acara Sekar Taman Rare saha paiketannyane ring wangun paajah-ajahan sakadi inucap ring sekolah manut Taksonomi Bloom. Wangun paajah-ajahan basa Bali sane kapaiketang ring tetujon pendidikan manut Taksonomi Bloom kapanggihin wangun paajah-ajahan sane madasar antuk wangun kognitif marupa paajah-ajahan tembang Bali inggihan Sekar Rare miwah Sekar Alit sane mawilang pat belas tembang. Wangun paajah-ajahan sane madasar antuk wangun afektif marupa paajah-ajahan sajeroning nilai pendidikan karakter. Wangun paajah-ajahan sane madasar antuk wangun psikomotor marupa kawagedan sajeroning matembang Bali inggihan Sekar Rare miwah Sekar Alit saha mabebaosan nganutin anggah-ungguhing basa Bali sane patut.

Paiketan wangun paajah-ajahan basa Bali sajeroning acara Sekar Taman Rare ring wangun paajah-ajahan sakadi inucap ring sekolah manut Taksonomi Bloom kapaiketang madasar antuk silabus paajah-ajahan basa Bali ring SD. Paiketan KAPUSTAKAN

Antara, I Gde Nala, dkk. 2016. Kamus Bali-Indonesia Beraksara Latin dan Bali Edisi II. Denpasar: Badan Pembina Bahasa, Aksara, dan Sastra Bali Provinsi Bali.

Arikunto, Suharsimi. 2006. Prosedur Penelitian: Suatu Pendekatan Praktik. Jakarta: Rineka Cipta

Bungin, Burhan. 2008. Penelitian Kualitatif: Komunikasi, Ekonomi, Kebijakan Publik, dan IImu Sosial Lainnya. Jakarta: Kencana

Creswell, John. 2015. Riset Pendidikan: Perencanaan, Pelaksanaan, dan Evaluasi Riset Kualitatif dan Kuantitatif. Yogyakarta: Pustaka Pelajar.

Djirne, I Wayan miwah I Wayan Roeme. 2000. Kumpulan Gending-Gending Bali. Denpasar: Cempaka. sane kapanggihin marupa paiketan sajeroning materi paajah-ajahan, paiketan sajeroning nilai pendidikan karakter, miwah paiketan sajeroning kawagedan matembang miwah mabebaosan. Paiketan sajeroning materi paajah-ajahan puniki kadasarin antuk wangun kognitif, paiketan sajeroning nilai pendidikan karakter kadasarin antuk wangun afektif, miwah paiketan sajeroning kawagedan matembang miwah mabebaosan kadasarin antuk wangun psikomotor.

Malarapan antuk pikolih sane sampun kabahbahang, prasida katurang piteket-piteket sakadi ring sor. Majeng ring Pangajah basa Bali mangda nguratiang acara sakadi Sekar Taman Rare puniki anggen nglimbakang wangun paajahajahan sane kamargiang ring sekolah.

Majeng ring Prodi Pendidikan Bahasa Bali mangda nglimbakang mata kuliah Tembang ngawit saking Sekar Rare santukan sampun wenten aturan indik paajah-ajahan ring SD patut kamargiang antuk pangajah basa Bali saking $S 1$ Pendidikan Bahasa Bali. Majeng ring Pemerintah Bali mangda nguratiang media massa ring Bali tur acara sakadi Sekar Taman Rare puniki sane pinaka silih sinunggil jalaran nglestariang basa Bali saha pinaka pendukung program pemerintah indik pelestarian basa Bali.

Gautama, I Wayan Budha. 2007. Kasusastraan Bali. Surabaya: Paramita

Hikmat, Mahi M. 2011. Metode Penelitian dalam Perspektif IImu Komunikasi dan Sastra. Yogyakarta: Graha IImu.

Holmes, Janet. 1992. An Introduction to Sociolinguistic. New York: Longman.

Kardiman, Yuyus. 2012. "Karakter adalah Akar Masalah Bangsa Kita". Jurnal Pendidikan, Volume 12 (kaca. 1728).

Mustari, Mohamad. 2011. Pengantar Metode Penelitian. Yogyakarta: LaksBang PRESSindo.

Nurgiyantoro, Burhan dan Anwar Effendi. 2013. "Prioritas Penentuan Nilai Pendidikan Karakter dalam Pembelajaran Sastra Remaja". Cakrawala Pendidikan Jurnal, 
Volume XXXII, Nomor 3(kaca. 113124).

Paramarta, I Ketut. 2009. Pemertahanan Bahasa Bali melalui Siaran Berbahasa Bali di BaliTV. Tesis (nenten kamijilang). Denpasar: Program Pasca Sarjana Universitas Udayana.

Peraturan Gubernur Nomor 80 Tahun 2018 Tentang Perlindungan dan Penggunaan Bahasa, Aksara, dan Sastra Bali serta Penyelenggaraan Bulan Bahasa Bali.

Putra, I Nyoman Darma. 2010. Tonggak Baru Sastra Bali Modern. Denpasar: Pustaka Larasan.

Soetantyo, Sylvia Primulawati. 2013. "Peranan Dongeng dalam Pembentukan Karakter Siswa Sekolah Dasar". Jurnal Pendidikan, Volume 14, Nomor 1 (kaca. 44-51).

Suandi, I Nengah dkk. 2016. Pedoman Penulisan Karya IImiah: Tugas Akhir, Skripsi, Tesis, dan Disertasi. Singaraja: Universitas Pendidikan Ganesha.

Suardika, I Wayan. 2015. Program Penyiaran Radio di Buleleng dalam Upaya Pemertahanan Bahasa Bali. Tesis (nenten kamijilang). Singaraja: Program Pasca Sarjana Universitas Pendidikan Ganesha.
Sujarweni, V. Wiratna. 2014. Metodologi Penelitian. Yogyakarta: Pustaka Baru Press.

Sukmadinata, Nana Syaodih. 2010. Metode Penelitian Pendidikan. Bandung: PT Remaja Rosdakarya Offset.

Sunarti dan Selly Rahmawati. 2014. Penilaian dalam Kurikulum 2013: Membantu Guru dan Calon Guru Mengetahui Langkah-langkah Penilaian Pembelajaran. Yogyakarta: Andi Offset.

Suparno, Paul. 2015. Pendidikan Karakter di Sekolah. Yogyakarta: PT. KANISIUS.

Syarbini, Amirulloh. 2012. Buku Pintar Pendidikan Karakter. Bandung: as@-prima.

Tinggen, I Nengah. 1994. Aneka Sari Gending-Gending Bali. Singaraja: Rhika Dewata.

Wirianti, Dewa Ayu Widi. 2017. Pemertahanan Basa Bali sajeroning Media Penyiaran ring Radio Citra Bali FM. Skripsi (nenten kamijilang). Singaraja: Universitas Pendidikan Ganesha.

Undang-Undang Dasar 1945

Undang-Undang Nomor 20 Tahun 2003 tentang Sistem Pendidikan Nasional 